\title{
Multi-frequency Backhaul analysis for UABS in disaster situations
}

\author{
German Castellanos* (D) \\ Dept. of Information \\ Technology \\ IMEC - Ghent \\ University \\ Ghent 9052, Belgium \\ german.castellanos@ugent.be
}

\author{
Margot Deruyck (i) \\ Dept. of Information \\ Technology \\ IMEC - Ghent \\ University \\ Ghent 9052, Belgium \\ margot.deruyck@ugent.be
}

\author{
Luc Martens (iD) \\ Dept. of Information \\ Technology \\ IMEC - Ghent \\ University \\ Ghent 9052, Belgium \\ luc1.martens@ugent.be
}

\author{
Wout Joseph (D) \\ Dept. of Information \\ Technology \\ IMEC - Ghent \\ University \\ Ghent 9052, Belgium \\ wout.joseph@ugent.be
}

\begin{abstract}
When a disaster occurs, the land-based cellular network could go offline for some days. Using an Unmanned Aerial Base Station (UABS) network is a promising solution to serve unconnected ground users. In this article, we propose a multifrequency backhaul architecture, which considers power and capacity constraints, to support the UABS network in a realistic 3D scenario in the city of Ghent, Belgium. Simulations results show that at the optimal flight height $(80 \mathrm{~m})$, up to $87 \%$ of the users could be supported using the multifrequency scenario compared with single frequency scenarios where coverage is about $70 \%$.
\end{abstract}

\section{Keywords-UABS, Backhaul, Disaster networks, UAV}

\section{INTRODUCTION}

Wireless cellular networks are quite reliable on a daily base. Nonetheless, when a disaster occurs like a hurricane or an earthquake, the cellular network can face difficulties such as saturation, when many people try to call, and the network is not able to manage such a peak of demanding traffic. Beside saturation also physical failure can be present when the infrastructure of towers, electricity and communication equipment is damaged. This was the case for the 2010 Haiti earthquake and the 2017 hurricane in Puerto Rico, where cellular communications were offline for two days [1], [2]. To solve this problem, a fast deployable network using Unmanned Aerial Vehicles (UAV) is widely studied [3]-[6]. In such a network, one base station is attached to an UAV that flies above the impaired area and provides voice and limited data access to the unconnected users. This kind of flying base station is called an Unmanned Aerial Base Station (UABS). To connect the UABSs with the core network an appropriate backhaul should be established. Several works have been addressing the UAV access network [5], [7]-[10], but the backhaul network is neglected so far.

In this study, we propose a multi-frequency backhaul architecture to serve the UABSs by using the $3.5 \mathrm{GHz}$ and $60 \mathrm{GHz}$ bands. To this end, we develop a deployment tool based on [11] that includes the proposed multifrequency backhaul network and apply it on a 3D realistic scenario in the city of Ghent, Belgium. To the best of the authors' understanding, a deployment tool for UABS networks, including backhaul connectivity is inexistent. The novelty of our approach is the mutual allocation of both the backhaul and access resources considering a multi-frequency backhaul connection, while optimizing capacity and power limitations.

The rest of this article is divided as follows. In Section II, the considered disaster circumstances and the UABS backhaul architecture are proposed. The description of the simulation tool and its network parameters is described in section III.

\footnotetext{
* German Castellanos is also affiliated to the Department of Electronics Engineering at Escuela Colombiana de Ingeniería. (Bogota, Colombia)
}

Section IV, presents the results of the multi-frequency backhaul networks and its analysis. Lastly, the article is closed in Section V with conclusions and future work.

\section{BACKHAUL NETWORK ARCHITECTURE FOR UABS IN DISASTER SITUATIONS}

\section{A. Multifrequency UABS Backhaul Architecture}

In [12], a general backhaul (BH) architecture for UAVaided network is introduced. A frequency of either $3.5 \mathrm{GHz}$ or $60 \mathrm{GHz}$ was selected for the backhaul connection due their advantages of low occupancy and licensed protection of these bands. However, only $72 \%$ and $69 \%$ of the users were covered when using the $3.5 \mathrm{GHz}$ and $60 \mathrm{GHz}$ bands, respectively. The main reason for the low user coverage is that all resource blocks (RB) in the backhaul were in use when using the $3.5 \mathrm{GHz}$ band, while path loss restrictions were the main problem when using $60 \mathrm{GHz}$.

To solve this problem, we propose a multifrequency backhaul architecture, as shown in Fig 1. When an emergency occurs, a truck filled with the UABSs drives to the center of the affected area and deploys the UAV-aided network. We will refer to this truck as the facility. The access network, specified in orange, provides voice and data connectivity to the ground users through $4 \mathrm{G}$ or $3 \mathrm{G}$ cellular technology using the $2.6 \mathrm{GHz}$ band. To provide backhaul connectivity to the deployed UAV network, the truck is equipped with a crane with an extensible arm that will raise an antenna to provide backhaul connectivity to UABSs. The backhaul proposed here is a multifrequency direct link that connects the UABSs with the core network through the antenna in the truck. The millimeter $60 \mathrm{GHz}$ is described in the purple arrow, while the sub-6GHz direct link is described in blue arrow. We use LTE (Long Term Evolution) technology, which helps to define the resource allocation parameters for the proposed architecture.

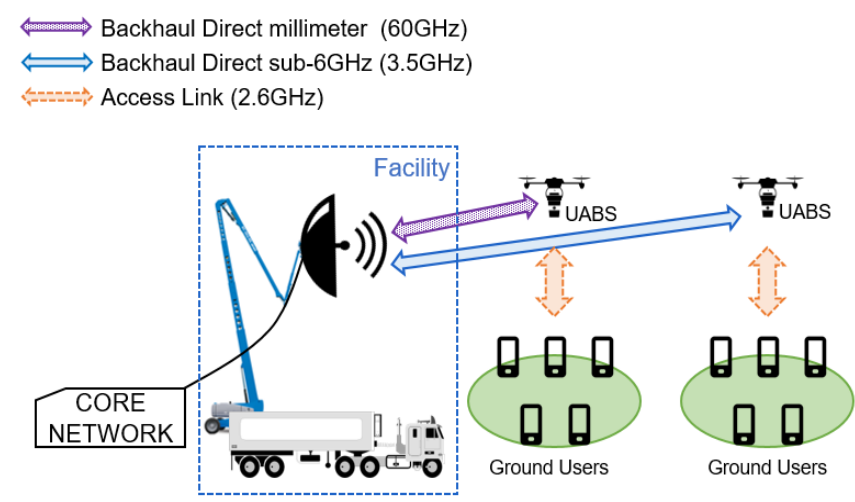

Fig. 1. Multifrequency backhaul architecture. 


\section{B. Backhaul Scenario definition}

We propose four different backhaul scenarios to serve UABSs. All of them have the same LTE femtocell access network using the $2.6 \mathrm{GHz}$ band. The first scenario (I) uses a $3.5 \mathrm{GHz}$ LTE network with a bandwidth (BW) of $20 \mathrm{MHz}$ (100RB). The second scenario (II) uses the $3.5 \mathrm{GHz}$ LTE network Carrier Aggregation (CA) in order to achieve a BW of $100 \mathrm{MHz}(500 \mathrm{RB})$. The third scenario (III) uses the mmwave band of $60 \mathrm{GHz}$ with a total of $9 \mathrm{GHz}$ of BW. The fourth scenario (IV) is a mixture between the II and the III scenarios. It uses either $60 \mathrm{GHz}$ or $3.5 \mathrm{GHz}$ bands to provide backhaul connectivity. The UABS first attempts to establish a $60 \mathrm{GHz}$ backhaul link, if it is not viable then tries with the $3.5 \mathrm{GHz} \mathrm{CA}$ link. The reason to try first the $60 \mathrm{GHz}$ is due it has available a huge number of RB (45000) compared with the $3.5 \mathrm{GHz}$ link, which it has only 500 RBs. A First-in-First-out (FiFo) algorithm is used to assign RBs to the UABSs' backhaul link concurrently to the access resource allocation process. In this article, we are going to focus on the IV scenario.

\section{Disaster Simulation StUdy}

\section{A. Disaster situation definition}

For the disaster situation, we assume that the whole existing cellular network is down, meaning that UABSs should support all the ground users. We use a realistic 3D model of the city center of Ghent $\left(6.85 \mathrm{~km}^{2}\right)$, Belgium, which includes the building blueprints. According to a Belgium mobile operator, 260 users are connected all together at peak hour [11]. Each user requires a bit rate of either 64kbps (voice call) or $1 \mathrm{Mbps}$ (data) and the users are uniformly distributed over the evaluated area. The user distribution parameters used in the simulation are presented in Table I. Three different types of drones to evaluate the performance of the backhaul network, as shown in Table II.

TABLE I. USERS DISTRIBUTION PARAMETERS FOR SIMULATION.

\begin{tabular}{|c|c|}
\hline Parameter & Value \\
\hline Ghent city size & $6.85 \mathrm{~km}^{2}$ \\
\hline Type of environment & Suburban \\
\hline Ground Users & 260 \\
\hline User location distribution & Uniform \\
\hline Traffic distribution & Voice $8.5 \% /$ Data $91.5 \%$ \\
\hline Traffic (Voice - Data) & $64 \mathrm{kbps}-1 \mathrm{Mbps}$ \\
\hline
\end{tabular}

TABLE II. TYPE OF DRONES FOR SIMULATION

\begin{tabular}{|c|c|c|c|}
\hline Drone Type & T1 & T2 & T3 Hybrid \\
\hline UAV battery capacity [Ah] & 2 & 17.33 & $100^{\text {a }}$ \\
\hline UAV battery voltage $[\boldsymbol{V}]$ & 14.3 & 22.2 & 48.0 \\
\hline UAV average usage $[\boldsymbol{W}]$ & 71.3 & 288.6 & 1200 \\
\hline Average UAV Speed $[\mathrm{m} / \mathbf{s}]$ & 15 & 12 & 15 \\
\hline Average Max Fight Time $[\mathbf{s}]$ & 900 & 2400 & 7200 \\
\hline
\end{tabular}

a. It includes the $37.5 \mathrm{Ah}$ generator with $1.5 \mathrm{l} / \mathrm{h}$ consumption and a 4 -liter tank.

\section{B. RF parameters}

The backhaul that we consider is a multifrequency LTE based network, which uses RB for capacity allocation as defined in the Orthogonal Frequency Division Multiple Access (OFDMA) of LTE. When using the $3.5 \mathrm{GHz}$ band, Carrier Aggregation (CA) is used to achieve a total of 500RBs [13]. The $60 \mathrm{GHz}$ band has a bandwidth of $9 \mathrm{GHz}$ equivalent to 45000 RBs. Table III presents the radio and link budget parameters used for this study. The 3GPP TR 38.901 [14] path loss model is considered for this study as proposed in [7]. The link budget parameters are shown in Table III.
TABLE III. BACKHAUL LINK BUDGET PARAMETERS

\begin{tabular}{|c|c|c|}
\hline Parameter & 3.5GHz Band & 60GHz Band \\
\hline Frequency & $3.5 \mathrm{GHz}$ & $61.5 \mathrm{GHz}$ \\
\hline Bandwidth & $100 \mathrm{MHz}$ & $9 \mathrm{GHz}$ \\
\hline \# of Resource blocks & 500 & 45000 \\
\hline Max Tx power & $43 \mathrm{dBm}$ & $10 \mathrm{dBm}$ \\
\hline BH Antenna Gain & $5 \mathrm{dBi}$ & $36 \mathrm{dBi}\left(2.5^{\circ}\right)$ \\
\hline Fade margin & $10 \mathrm{~dB}$ & $5 \mathrm{~dB}$ \\
\hline $\begin{array}{l}\text { Receiver Signal-to-Noise } \\
\text { Ratio (SNR) for } \\
\text { Modulation and Coding } \\
\text { Scheme (MCS) }\end{array}$ & $\begin{array}{c}1 / 3 \text { QPSK }=-1.5 \mathrm{~dB} \\
1 / 2 \mathrm{QPSK}=3 \mathrm{~dB} \\
2 / 3 \mathrm{QPSK}=10.5 \mathrm{~dB} \\
1 / 216-\mathrm{Q} A M=14 \mathrm{~dB} \\
2 / 316-\mathrm{Q} \mathrm{MM}=19 \mathrm{~dB} \\
1 / 264-\mathrm{QAM}=23 \mathrm{~dB} \\
2 / 364-\mathrm{QAM}=29.4 \mathrm{~dB}\end{array}$ & $\begin{array}{c}1 / 2 \mathrm{BPSK}=7.39 \mathrm{~dB} \\
1 / 2 \mathrm{QPSK}=15.4 \mathrm{~dB} \\
1 / 216 \mathrm{QAM}=17.5 \mathrm{~dB}\end{array}$ \\
\hline Noise figure in UABS & \multicolumn{2}{|c|}{$5 \mathrm{~dB}$} \\
\hline Shadowing margin & \multicolumn{2}{|c|}{$8.2 \mathrm{~dB}$} \\
\hline Facility antenna height & \multicolumn{2}{|c|}{$25 \mathrm{~m}$} \\
\hline
\end{tabular}

\section{Simulation tool}

To evaluate the backhaul architecture proposed, we develop a Java tool based as an extension of the one used in [11] and [12]. Here, the addition of the backhaul resource allocation concurrently with the access resource allocation allows controlling the power and capacity limitations of the network. The novelty of this study is the inclusion of the multifrequency radio resource allocation as described next.

The algorithm to design the backhaul network is as follows. First, the traffic and user distributions and the facility location are generated. Second, for each user, a list of feasible UABSs to connect with is determined. To evaluate if a UABS is a possible candidate, the algorithm first calculates if the $60 \mathrm{GHz}$ link is viable in terms of path loss. If the $60 \mathrm{GHz}$ is not feasible, then the $3.5 \mathrm{GHz} \mathrm{CA}$ is evaluated in the same way. Third, the first link that fulfils the requirements is assessed in terms of capacity. The link evaluation includes the number of $\mathrm{RB}$ demanded by a user connection in the backhaul link by a given Modulation and Coding Scheme (MCS) defined by the signal to noise ratio (SNR) in link budget. Fourth, the user is allocated to that UABS and capacity values are updated. Finally, the algorithm finishes when all users are served or when the RBs are fully used.

\section{RESULTS}

\section{A. Multifrequency Scenario}

First, we evaluate the performance of the network by evaluating the fly elevation of the UABSs. We variate the altitude from 20 to $200 \mathrm{~m}$ looking for an optimal elevation. In Fig. 2, we present the results of the number of served users (in bars) with the three types of drones and the used locations of the UABS (in lines). The results show that the optimal altitude is at $80 \mathrm{~m}$, when the maximum number of served users $(225$ for type 1 drone, 227 and 221 for type 2 and 3 respectively) is accomplished. The performance of the three types of drones is similar because the way our tool assigns the UABS locations is based on the position of the served users and not based on the type of drone used. In the continued lines of Fig. 2, the number of UABS locations is shown. It could be seen that the UABS' locations increase rapidly until $60 \mathrm{~m}$, with a peak of 26 UABSs, then decrease slowly to 14 UABSs at $200 \mathrm{~m}$ fly elevation. This behavior is related to the footprint of the city of Ghent and the path loss. The lower the drone flies, the more non-Line-of-Sight (NLoS) links are present and thus, fewer UABSs are connected. As altitude increases, more LoS links emerge and more UABSs could connect, serving more users. If the fly height increases, the path loss will be better and the 
number of UABS will decrease. The highest ratio of served users per UABS, around 8.7 users per UABS, is obtained at $80 \mathrm{~m}$. Similar numbers are also seen above $180 \mathrm{~m}$ but are not considered because the total number of served users by the whole UABS network is smaller than at $80 \mathrm{~m}$. Furthermore, these altitudes are also above the maximum fly height allowed by the Belgian regulation authorities.

The total number of used UABSs is presented by the bars of Fig. 3. Here it can be seen that almost four times more type 1 drones are required than for type 2 and 3, due to the maximum flight time described in Table II. Despite that type 2 and 3 drones have different maximum flight time (nearly three times larger than type 1), the service time for both types is close to one hour leading to fewer drones being used. Comparatively, the lines in Fig. 3 present the average consumption per UABS. Here, consequently, the power consumption of the type 1 drone, with an average of $7.7 \mathrm{~W}$ per UABS, is 4.5 times lower than type 2 and 3 . Type 2 and 3 consume an average of $33.3 \mathrm{~W}$ and $36.2 \mathrm{~W}$, respectively. The hybrid drone consumes a little more because it has to account for the extra weight of the fuel tank and the generator.

The capacity used by the backhaul is presented in Fig. 4 . Similar to the number of served users, the maximum capacity i.e., a total of $206.6 \mathrm{Mbps} \pm 1.2 \%$, is obtained at $80 \mathrm{~m}$ fly altitude as shown in Fig 4.a. Fig 4.a also shows the available capacity for each used backhaul frequency $(60 \mathrm{GHz}$ and $3.5 \mathrm{GHz} \mathrm{CA})$. As fly height increases, the demanded traffic increases with a top at $80 \mathrm{~m}$. Particularly, the $60 \mathrm{GHz}$ link increases changing for an average of $12.9 \%$ to a top of $26 \%$ at $80 \mathrm{~m}$ fly height. This behavior is clarified in Fig 4.b, where the number of resource blocks used by the $3.5 \mathrm{GHz} \mathrm{CA}$ technology (dotted lines) is fully used from $40 \mathrm{~m}$ fly height. Hence, $60 \mathrm{GHz}$

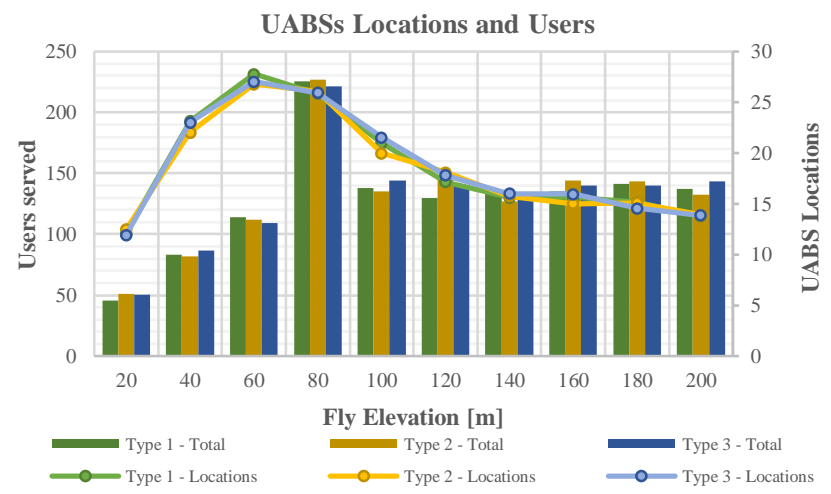

Fig. 2. Multifrequency scenario served users and UABS locations for different fly elevation.

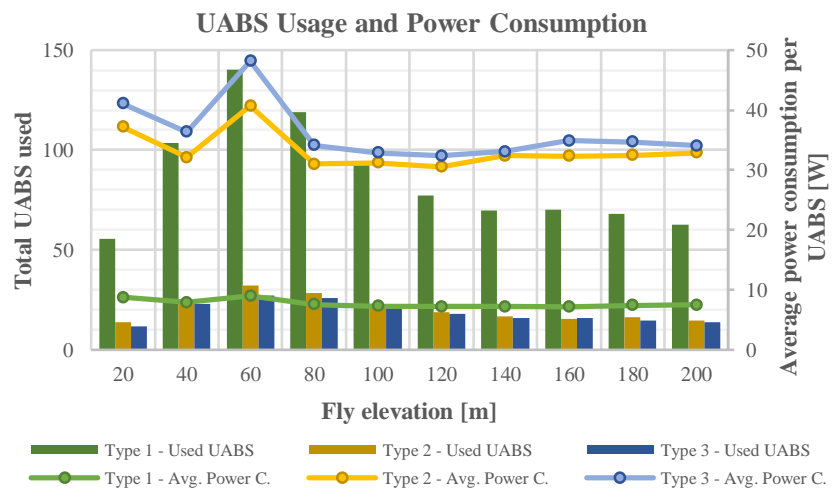

Fig. 3. Multifrequency scenario served users and UABS locations for different fly elevation. connections have to support the remaining demanding traffic. The difference between the ratio of capacity and the number of used RB in each technology is based on the spectrum efficiency of the MCS described in Table III.

Next, we evaluate the performance of the network when the demanding ground users vary. We change the number from 50 to 500 users to evaluate the performance of the network. In Fig. 5, the number of users served is presented. The lines described the percentage of users served compared with the demanding users. As shown in Fig. 5, a maximum of about 230 users can be served by the UABS network. The maximum number of served users by the different types of drones are quite similar, changing in ranges of $2 \%$ among them. The reason why no more user can be covered can be found in Fig. 6 which presents the network capacity for a varying number of demanding users. The total number of UABS connected to the core network is presented in Fig. 6.a. The more users we want to cover, the more UABS are required. Indeed, the total number increases but only up to 230 users. For higher values, it is no longer possible to connect the extra required UABSs to the backhaul network. The dotted lines in Fig. 6a correspond to the $3.5 \mathrm{GHz} \mathrm{CA}$ technology, which allows connecting a maximum of 20 UABSs. This corresponds with a capacity of around $155 \mathrm{Mbps}$ as shown in Fig. 6.b. The reason for this could be found when we examine the number of used resource blocks per technology (dotted lines in Fig. 6.c). Here it is shown that for more than 200 users, the number of used $\mathrm{RBs}$ in the $3.5 \mathrm{GHz} \mathrm{CA}$ obtained the maximum of 500RBs. As a consequence, the $60 \mathrm{GHz}$ tries to compensate the demand not covered by the $3.5 \mathrm{GHz}$ CA links.

In Fig 6.b, it can be seen that for the $60 \mathrm{GHz}$ links (dashed lines) the capacity increases slightly after 250 demanding users, but it is not sufficient to serve UABS that flies far away from the facility antenna.

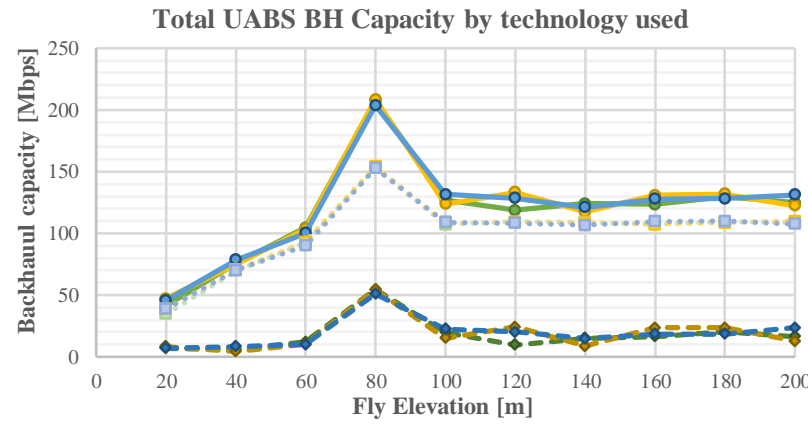

(a)

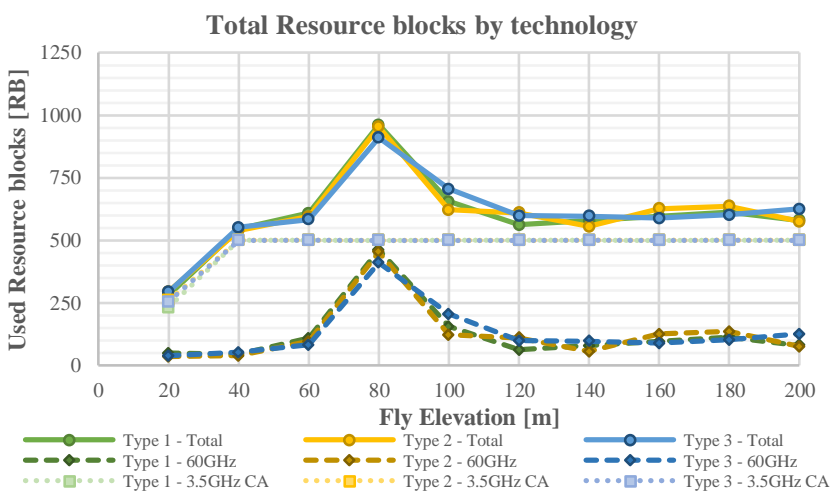

(b)

Fig. 4. Capacity evaluation. (a) Backhaul traffic. (b) Resource block used 


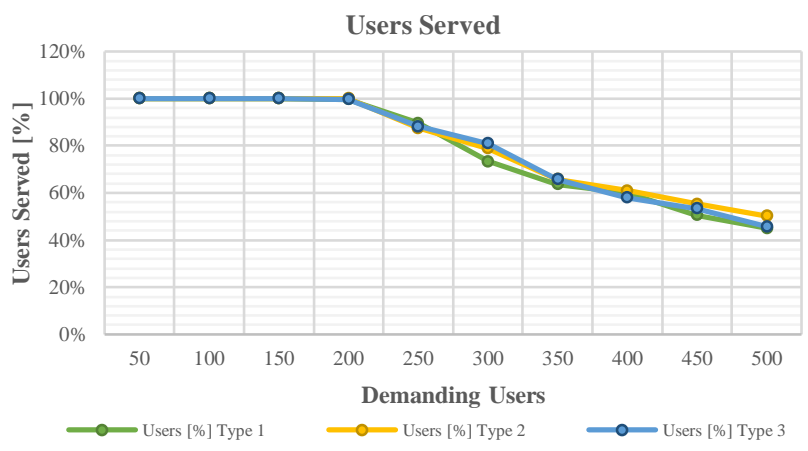

Fig. 5. Multifrequency scenario served users for different demanding users

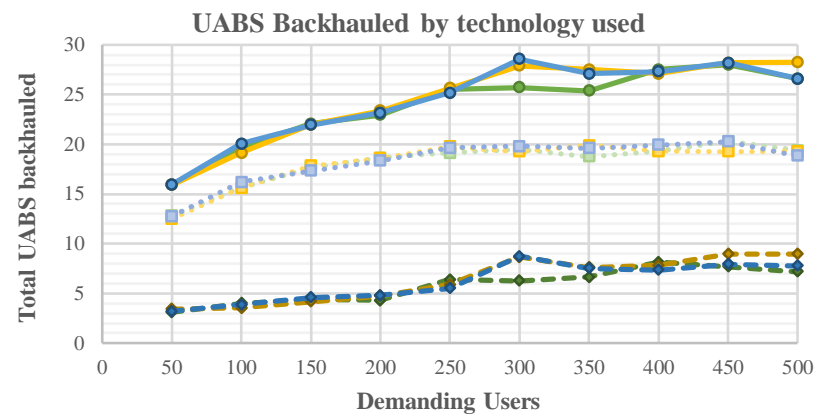

(a)

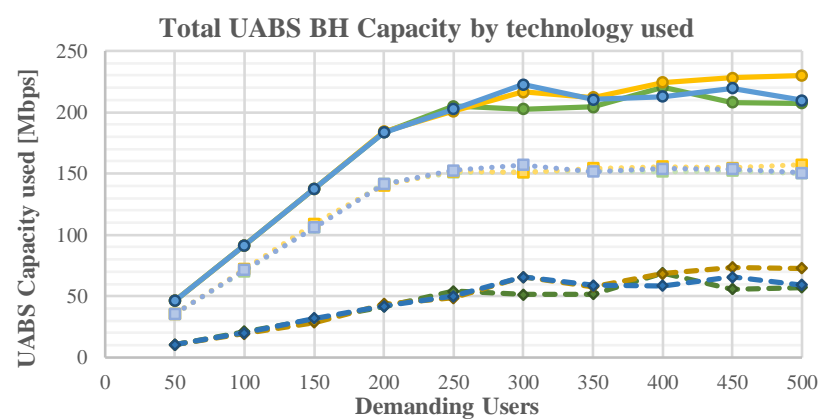

(b)

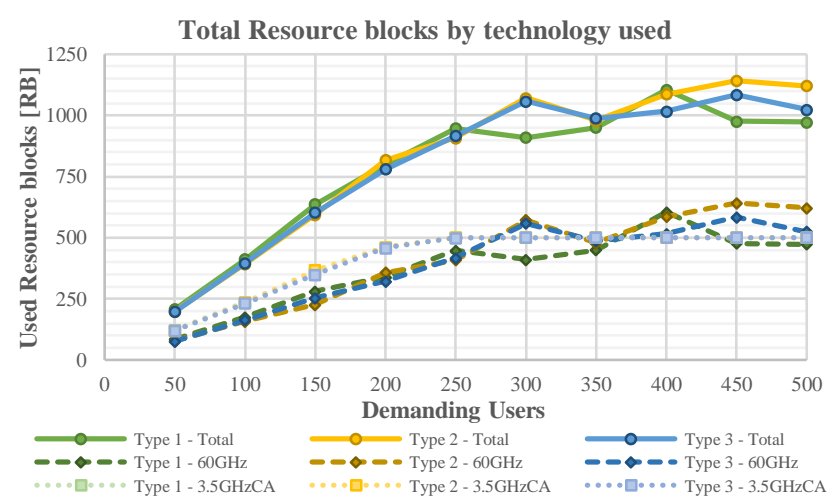

(c)

Fig. 6. Multifrequency scenario capacity study. (a) Total number of UABS backhauled. (b) Total traffic used. (c) Total resource blocks used.

\section{B. Comparison with Single frequency scenarios}

In this section, we will compare the three single frequency scenarios $(3.5 \mathrm{GHz}$ - Scenario I, 3.5GHz CA - Scenario II, or $60 \mathrm{GHz}$ - Scenario III) proposed in [12] with our multifrequency scenario (combining $3.5 \mathrm{GHz} \mathrm{CA}$ and $60 \mathrm{GHz}$ for backhauling - Scenario IV). In Fig. 7, we compare the number of users served for the four scenarios when varying the number of demanding users. Here, it can be seen that for the two $3.5 \mathrm{GHz}$ scenarios the number of served users is stabilized at 54 and 188 users without and with CA respectively. In both scenarios, the resource blocks are fully used, as shown by the green and blue bars in Fig. 7. Scenario II is able to support up to 200 users. For Scenario III, using only a $60 \mathrm{GHz}$ link, the system is able to connect only between $55 \%$ and $70 \%$ of the demanding users, because if $60 \mathrm{GHz}$ is used, far distant UABS could not connect and in consequence, far distant ground users are felt unserved. This is the case at 500 demanding users, the system is able to serve around the 350 closest, leaving the 150 far distant unserved. Although we can still not cover all users above a user demand of 200, the multifrequency configuration of Scenario IV can serve more users compared to Scenario II. This is because when the $3.5 \mathrm{GHz} \mathrm{CA}$ link is fully used, the $60 \mathrm{GHz}$ is available to connect some extra UABSs to serve more users as shown by the brown line in Fig. 7. In fact, it is even possible to slightly increase the user coverage percentage when increasing the demanding user since due to the uniform distribution of the users, a larger number of users will be inside the $60 \mathrm{GHz}$ coverage area. Note that Scenario III outperforms the multifrequency scenario for more than 350 demanding users in terms of served users. However, this does not imply that far away users are being served, as it is the case in the multifrequency scenario. The multifrequency approach is fairer because the whole coverage area is bigger.

In order to present a full comparison of the disaster situation, we present in Table IV, a resume of the results for the more critical parameters in our network for the optimal altitude of $80 \mathrm{~m}$ and 260 ground users. The first parameter is the number of users. We can see that for this situation, the multifrequency scenario serves $18.8 \% \pm 2 \%$ and $26.6 \% \pm 1 \%$ more users than Scenario II and III, respectively. Second, we consider the parameter UABS locations. In the multifrequency scenario $10 \%$ and $37 \%$ more UABS locations are served than for Scenario II and III, respectively. Moreover, the power efficiency is better in Scenario III with $139 \mathrm{~mW}$ per served user, while Scenario IV has an average of $153.7 \mathrm{~mW}$ per served user. This is due to the fact that the maximum allowed input power in the $60 \mathrm{GHz}$ technology is $33 \mathrm{~dB}$ less than $3.5 \mathrm{GHz}$ technology. Finally, the capacity served by scenario IV is significantly higher, over $203 \mathrm{Mbps}$, than for the other scenarios. However, the capacity of each RB is smaller compared to all the $3.5 \mathrm{GHz}$ scenarios. Overall, the performance of scenario IV is slightly better than scenario II but quite similar. The reason for this is that nearly threequarters of the network are served by $3.5 \mathrm{GHz} \mathrm{CA}$ technology, hence their similar behavior.

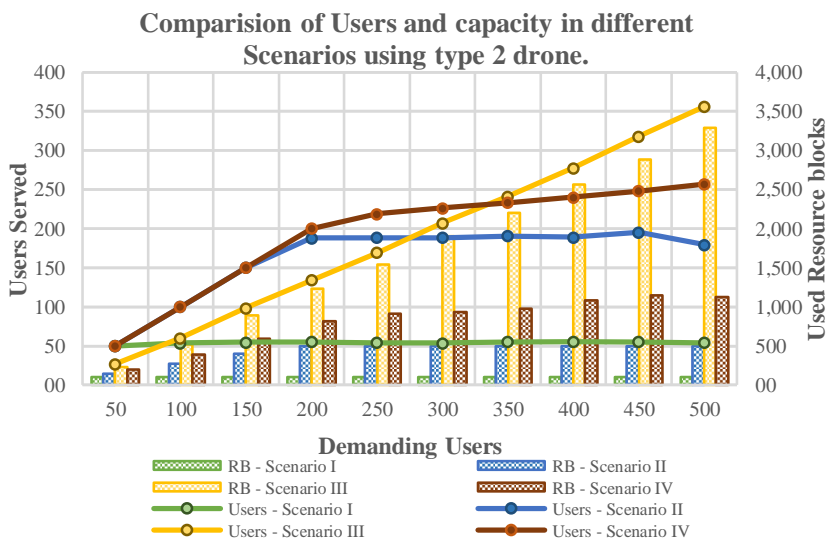

Fig. 7. Comparison of users served and capacity for different scenarios. 
TABLE IV. COMPILATION OF MAIN PARAMETERS FOR THE FOUR SCENARIOS WITH 260 USERS AND 80M FLIGHT ALTITUDE

\begin{tabular}{|c|c|c|c|c|c|c|c|c|c|c|c|c|}
\hline & \multicolumn{3}{|c|}{ Scenario I } & \multicolumn{3}{|c|}{ Scenario II } & \multicolumn{3}{|c|}{ Scenario III } & \multicolumn{3}{|c|}{ Scenario IV } \\
\hline & Type 1 & Type 2 & Type 3 & Type 1 & Type 2 & Type 3 & Type 1 & Type 2 & Type 3 & Type 1 & Type 2 & Type 3 \\
\hline & \multicolumn{12}{|c|}{ USERS } \\
\hline Users served [users] & 54 & 54 & 54 & 188 & 188 & 191 & 178 & 178 & 178 & 225 & 227 & 221 \\
\hline Users served [\%] & 21.6 & 21.7 & 21.7 & 72.4 & 72.3 & 73.4 & 68.3 & 68.5 & 68.3 & 86.6 & 87.3 & 85.2 \\
\hline Users per UABS & 3.36 & 3.2 & 3.4 & 8.1 & 7.9 & 8.1 & 9.4 & 10.1 & 9.6 & 8.7 & 8.7 & 8.6 \\
\hline \multicolumn{13}{|c|}{ UABSS } \\
\hline \# UABSs Locations & 16.4 & 17.0 & 16.2 & 23.4 & 23.9 & 23.6 & 19.2 & 18.6 & 18.8 & 25.8 & 26.1 & 25.9 \\
\hline \# Used UABS & 75.2 & 18.5 & 16.2 & 110.1 & 26.5 & 23.6 & 76.7 & 18.6 & 18.8 & 119.1 & 28.5 & 25.9 \\
\hline Mean Power Usage [w] & 34.4 & 33.7 & 35.3 & 36.9 & 36.2 & 36.9 & 24.9 & 24.9 & 24.9 & 35.1 & 33.9 & 34.4 \\
\hline \multicolumn{13}{|c|}{ CAPACITY } \\
\hline Total BH Capacity [Mbps] & 47.4 & 47.6 & 47.9 & 173.1 & 171.4 & 174.7 & 163.8 & 164.1 & 163.5 & 207.6 & 208.6 & 203.7 \\
\hline BH Capacity / UABS [Mbps] & 2.9 & 2.8 & 2.9 & 7.4 & 7.2 & 7.4 & 8.7 & 8.8 & 8.8 & 8.1 & 8.6 & 7.9 \\
\hline Total RB Usage [RB] & 100 & 99.9 & 100 & 500 & 499.8 & 499.9 & 1617.8 & 1619.1 & 1620.8 & 961.7 & 952.2 & 909.6 \\
\hline RB Usage per UABS [RB] & 6.2 & 5.9 & 6.2 & 21.5 & 21.0 & 21.3 & 85.4 & 87.1 & 87.36 & 37.3 & 36.6 & 34.9 \\
\hline RB Capacity [kbps/RB] & 474.0 & 476.5 & 479.0 & 346.2 & 342.9 & 349.5 & 101.2 & 101.3 & 100.8 & 217.1 & 234.9 & 226.4 \\
\hline BH RB Efficiency [\%] & 87.0 & 87.2 & 87.1 & 96.2 & 96.4 & 96.1 & 98.4 & 98.7 & 98.5 & 97.2 & 96.9 & 97.1 \\
\hline
\end{tabular}

\section{CONCLUSIONS AND FUTURE WORK}

In this study, we introduced a multifrequency direct link backhaul architecture to support ground users in a disaster situation. This scenario considers the $3.5 \mathrm{GHz}$ and $60 \mathrm{GHz}$ bands to connect UABSs to a central transportable facility. To evaluate the performance of the architecture, we developed a Java based tool to simulate the network based on real information from a Belgian operator and a 3D realistic model from the city of Ghent in Belgium. The novelty of the proposal is the conjoint allocation of backhaul and access radio resources based on capacity and power restrictions in the UABS. The results show that the described architecture is able to support up to $86.4 \% \pm 1 \%$ of the ground users in the disaster situation compared to a maximum of $72 \% \pm 1 \%$ for the single frequency Scenario II in Table IV. The results also show that only 26 UABS are needed to support 221 users in the best case scenario at an optimal fly altitude of $80 \mathrm{~m}$. The main limitation to have full coverage is the capacity restriction of the $3.5 \mathrm{GHz}$ CA technology, where all of the 500 RBs are fully used. Moreover, despite that the $60 \mathrm{GHz}$ technology has a vast number of $\mathrm{RB}$, the path loss constraints limit the connection of distant UABSs and in consequence, distant users from the facility center. The usage of multifrequency technology allows a fair allocation of ground users over the complete disaster area.

Despite the coverage is nearly $87 \%$ of the disaster area, if more ground users are demanding connectivity network performance decreases. To solve this, future work will be focused on multihop connectivity to take advantage of the vast bandwidth of the $60 \mathrm{GHz}$ band and the versatility of the sub$6 \mathrm{GHz}$ in-band backhauling. Also, the usage of MIMO, beamforming and better scheduling schemes will be proposed for improvements to the proposed architecture.

\section{ACKNOWLEDGMENTS}

G. Castellanos is Funded by COLFUTURO and Colombian School of Engineering. (Bogota, Colombia)

M. Deruyck is a Post-Doctoral Fellow of the FWO-V (Research Foundation - Flanders).

\section{REFERENCES}

[1] 'Statement From Digicel on Haiti Earthquake', 20-Aug-2010. [Online]. Available: https://web.archive.org/web/20100820123624/http://www.indiaprwir e.com/pressrelease/telecommunications/2010011441347.htm. [Accessed: 19-Jun-2019].

[2] '2017 Hurricane Season FEMA After-Action Report', p. 65, 2017.

[3] N. Zhao et al., 'UAV-Assisted Emergency Networks in Disasters', IEEE Wireless Communications, vol. 26, no. 1, pp. 45-51, Feb. 2019.

[4] C. T. Cicek, T. Kutlu, H. Gultekin, B. Tavli, and H. Yanikomeroglu, 'Backhaul-Aware Placement of a UAV-BS with Bandwidth Allocation for User-Centric Operation and Profit Maximization', arXiv:1810.12395 [cs, math], Oct. 2018.

[5] Y. Zeng, J. Lyu, and R. Zhang, 'Cellular-Connected UAV: Potential, Challenges, and Promising Technologies', IEEE Wireless Communications, vol. 26, no. 1, pp. 120-127, Feb. 2019.

[6] A. V. Savkin and H. Huang, 'Deployment of Unmanned Aerial Vehicle Base Stations for Optimal Quality of Coverage', IEEE Wireless Communications Letters, vol. 8, no. 1, pp. 321-324, Feb. 2019.

[7] M. Deruyck, A. Marri, S. Mignardi, L. Martens, W. Joseph, and R. Verdone, 'Performance evaluation of the dynamic trajectory design for an unmanned aerial base station in a single frequency network', in Proceedings of the IEEE 28th International Symposium on Personal, Indoor and Mobile Radio Communications, 2017, pp. 1-7.

[8] A. Merwaday and I. Guvenc, 'UAV assisted heterogeneous networks for public safety communications', in 2015 IEEE Wireless Communications and Networking Conference Workshops (WCNCW), 2015, pp. 329-334.

[9] S. A. W. Shah, T. Khattab, M. Z. Shakir, and M. O. Hasna, 'A Distributed Approach for Networked Flying Platform Association with Small Cells in 5G+ Networks', 2017, pp. 1-7.

[10] M. Mozaffari, W. Saad, M. Bennis, and M. Debbah, 'Efficient Deployment of Multiple Unmanned Aerial Vehicles for Optimal Wireless Coverage', IEEE Communications Letters, vol. 20, no. 8, pp. 1647-1650, Aug. 2016.

[11] M. Deruyck, J. Wyckmans, W. Joseph, and L. Martens, 'Designing UAV-aided emergency networks for large-scale disaster scenarios', EURASIP Journal on Wireless Communications and Networking, vol. 2018, no. 1, Dec. 2018.

[12] G. Castellanos, M. Deruyck, L. Martens, and W. Joseph, 'Performance Evaluation of Direct-Link Backhaul for UAV-Aided Emergency Networks', Sensors, vol. 19, no. 15, p. 3342, Jan. 2019.

[13] D. Carrillo, F. A. P. de Figueiredo, F. L. Figueiredo, and J. P. Miranda, 'LTE and Beyond', in Long Term Evolution: $4 G$ and Beyond, A. Paradisi, M. D. Yacoub, F. Lira Figueiredo, and T. Tronco, Eds. Cham: Springer International Publishing, 2016, pp. 1-25.

[14] ETSI, 'ETSI TR 138901 v14.3.0 - 5G; Study on channel model for frequencies from 0.5 to $100 \mathrm{GHz}$ (3GPP TR 38.901 version 14.3 .0 Release 14)', Jan. 2018. 